\title{
VARIANT OF ACCESSORY QUADRATE LOBE AND FISSURES IN THE LIVER AND THEIR CLINICAL CORRELATION
}

\author{
Iv. Maslarski* \\ Departmant of Anatomy, Histology, Pathology and Forensic Medicine, Faculty of Medicine, \\ SU "St. Kliment Ohridski", Sofia, Bulgaria
}

\begin{abstract}
There are numerous variations in the human body, especially when considering blood vessels or peripheral nerves. Regarding the liver, the variations tend to occur in the vessels or ducts, whereas variations with the liver lobes are a rare phenomenon. The presence of an additional lobe or different compartments outside the norm can be due to embryological heteroplasia, which has a congenital origin. They can also be a consequence of a surgical intervention, compression of neighbouring organs or an increased volume of the diaphragm. Sometimes there are cases where other enlargements are mistaken for extra liver compartments for example enlarged lymph nodes, presence of liver cysts or haematoma or even build-up of fluids in the region of certain fissures.

This finding was discovered in a routine dissection with medical students in Medical Faculty of SU "St. Kliment Ohridski". What can be seen is accessory quadrate lobe (AQL), some fissures or sulci on the anterosuperior surface of the liver.

The case mentioned above, is a rare phenomenon, as it occurs in under $10 \%$ of the human population and has a clinical significance regarding: diagnosis, surgery and transplantation. Awareness of this variation type will help avoid diagnostic errors and avoid complications or a fatal outcome.
\end{abstract}

Key words: liver variations, fissures, additional liver lobe, agenesis

\section{INTRODUCTION}

The liver is the largest gland in the human body. Its external morphology is characterized by an irregular shape and the presence of lobes, which are limited by clearly defined anatomical structures (ligaments, vessels, gallbladder).

Liver variations associated with an absence of lobe or presence of an additional liver lobe are quite rare. Congenital abnormalities of this organ are quite rare, especially when compared with the presence of inherent pathologies of other organs. Nowadays liver transplantations have become more successful due to modern medical imaging and new surgical techniques. Liver surgery, bile, biliary tract, as well as

\footnotetext{
*Correspondence to: Ivan Maslarski, Departmant of Anayomy, histology, pathology and forensic medicine - Faculty of medicine, SU "St. Kliment Ohridski”,1407 Sofia, 1 Kozyak str., Bulgaria, Email:maslarsky@gmail.com,Phone: +359882000809
}

liver transplantation, depend on the morphological variations of this parenchymal organ. Any significant variation of the norm deserves to be described and promoted in the medical literature.

\section{MATERIAL AND METHODS}

Such finding have been noted in a routine dissection of the upper and middle area of the abdomen with medical students in the Medical Faculty of University of Sofia "St. Kliment Ohridski". The cadaver has been fixed with standard solution containing formalin. After removal of the organ located more in the right hypochondrium and epigastrium and also extending considerably into the left hypochondrium till the left lateral line, the morphological variation was noted.

\section{RESULTS}

During the past ten years 20 cadavers has been dissected with an age ranging between 62 and 70 years old ( $80 \%$ of whom were female) at the gross anatomy lab of the University of 
Sofia "St. Kliment Ohridski". All cadavers were of Bulgarian origin, with no associated gross pathological changes or presence of surgical scars.

A clinical case had been detected in a 62 year old female corpse with unusual morphology on the dorsal surface of the liver. A surface with a deep groove in the area of porta hepatis was noted. What can be seen is accessory quadrate lobe (AQL), some fissures or sulci on the anterosuperior surface of the liver (Figures 1-
MASLARSKI IV.

3). In regards to other morphological characteristics, no variations have been observed, either in metric indicators or in the position of the ligaments, gallbladder and bile pathways. Blood vessels complies within the norm. The topographic position of the organ was in accordance with the anatomical nomenclature. The shape of the largest gland in the human body is triangular with its dimensions of length $-3.7 \mathrm{~cm}$ from its apex to base, thickness $0.7 \mathrm{~cm}$ and base $-1.5 \mathrm{~cm}$.

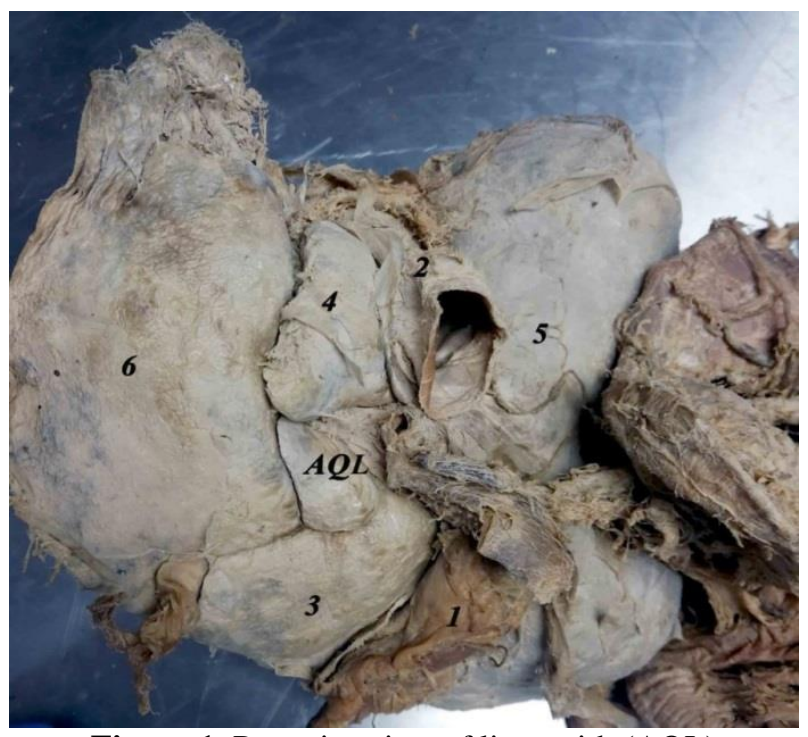

Figure 1. Posterior view of liver with (AQL)

AQL - accessory quadrate lobe; 1 . gallbladder; 2 . superior vena cava; 3 . quadrate lobe; 4. caudate lobe; 5 . right lobe; 6 . left lobe.

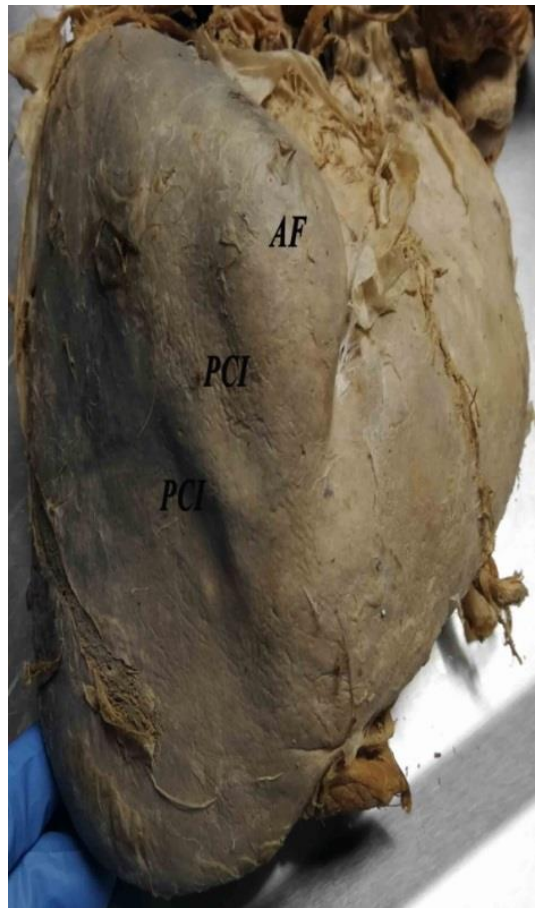

Figure 2. Accessory fissure (AF) on anterior surface of liver

$\mathrm{AF}$ - accessory fissure; PCI - prominent costal deep impressions.

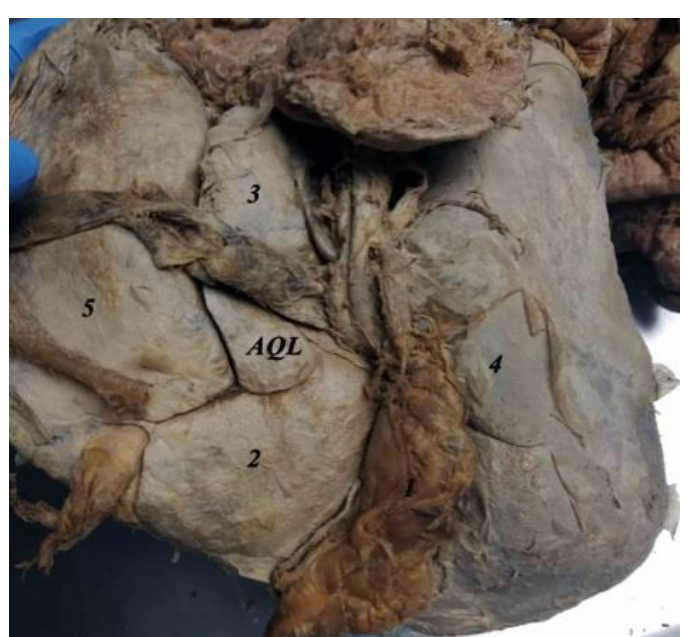

Figure 3. Another view of accessory quadrate lobe 1.gallbladder; 2.quadrate lobe; 3.caudate lobe; AQL - accessory quadrate lobe; 4.right lobe; 5.left lobe

\section{DISCUSSION}

The regular liver morphology has a triangular shape based on (regarding is also acceptable) the rapid change in diameter in the upper and lower directions and from right to left. The main macroscopic structures of the anterior Trakia Journal of Sciences, Vol. 17, Suppl. 2, 2019 
MASLARSKI IV.

surface are two lobes, which are separated by a falciform ligament. The posterior view of the liver is anatomically represented by four lobes separated by venosum ligament, teres ligament, cava inferior vein and gallbladder. Porta hepatis is located between these structures which appear like the letter $\mathrm{H}$. The posterior lobes are left, right, quadrate and caudal.
This particular case report cannot be statistically significant, since the dissected cadavers in the time period (previously mentioned) are below 100, which is the number required for quantitative analysis. We will refer to data on frequent liver morphological variations from different authors' teams in Table 1.

Table 1. Classification of liver according to Netter's

\section{Netter type}

No of

specimens

Type 1- very small left lobe, deep costal impressions.

Type 2- complete atrophy of left lobe

Type 3- transverse saddle like liver with relatively large left lobe

Type 4- tongue like process of right lobe(Reidel's lobe)

Type 5- very deep renal impression \& corset constriction

Type 6- diaphragmatic grooves
$1(2 \%)$

0

$2(4 \%)$

$1(2 \%)$

$3(6 \%)$

$2(4 \%)$
Variations of the liver in terms of external morphology are a relatively rare phenomenon and there is not enough sources identifying such variations [2]. As we have noted earlier, this is not the case for blood vessels. Quite often the variations are due to different congenital abnormalities, such as agenesis or immature lobes, segments of the liver. Other characteristic variations is the absence of segments, reduced lobe size, hypoplastic lobes, repositioning of the gallbladder and additional.

Sulci and Fissures along the anterolateral surface are most commonly found in liver variations. As it can be seen in our case in Figure 1, an additional structure was observed, which according to Macchi and Auch [1,5,6], results from uneven growth of the liver parenchyma. Bundles of diaphragm muscle shows different resistance and this results in different compression of the parenchyma, which and as a results in additional groovs and folds. Another theory for the occurrence of these variable structures results due to the socalled weak areas in the parenchyma, which cannot counteract the muscular compression of the contraction of the diaphragm muscle and leads to such relief. These statements are supported by research using a variety of medical imaging.
Additional lobes, such as the case described in Figure 2, are relatively rare and are a matter of interest. In our case, there is an additional quadrate lobe. It is not clear what causes this appearance, usually it is a quadrate lobe containing a physical fissure that divides the lobe in two parts. According to other authors $[3,4,7]$, the extra hepatic tissue is connected to the main organ, although there are cases where it is completely separated from the normal liver.

\section{CONCLUSIONS}

The external morphological hepatic variations (not related to vascular nerve bundles) are often underestimated. We believe that their description and promotion in combination with current medical imaging will lead to more successful liver surgeries. Moreover, intraoperative ultrasonography is commonly used in liver surgery to determine the rate of lesion and the knowledge of organ morphological variations will prove to be of high importance.

Hepatic variations can have clinical implications, therefore prove to be more than an "uncharacteristic external morphology". The Identification of such variations while still living, through various medical imaging techniques of diagnosis, is an important source 
for analyzing what they are derived from. It is a common practice to ignore them which on the otherhand leads to poor clinical outcome. Evidently a more detailed study of the liver variations in combination with the causes leading to this condition will be needed in the future. Such cases are important for medical imaging and general surgery professionals to improve diagnosis and to plan appropriate surgery.

\section{REFERENCES}

1. Auh, YH., Rubenstein, WA., Zinnsky, K., et al., Accessory fissures of the liver: CT and sonographic appearance. Am $J$ Roentgenol, 143:565-72, 1984.(3)

2. Chin, J., O'Toole,P.,et al., Hepatic morphology: variations and its clinical
MASLARSKI IV.

importance. Eur. J. Anat., 22 (3): 195-201, 2018.

3. Collan, Y., Hakkiluoto, A., Hastbacka, J., Ectopic liver. Ann Chir Gynaecol ,67:2729, 1978.(4)

4. Kostov, DV., Kobakov, GL., A large accessory liver lobe. HPB (Oxford), 13(10):757, 2011.

5. Macchi, V., Feltrin, G. Parenti, A., De Caro,R., Diaphragmatic sulci and portal fissures. J Anat.,202:303-8, 2003.(1)

6. Macchi, V., Porzionato, A., Parenti,A., et al., Main accessory sulcus of the liver. Clin Anat., 18:39-45, 2005.(2)

7. Rutkauskas, S., Gedrimas, V., et al., Clinical and anatomical basis for the classification of the structural parts of liver. Medicina (Kaunas)42(2): 98-106, 2006. 